\section{PMO-239 INFLAMMATORY BOWEL DISEASE AND PREGNANCY: LACK OF KNOWLEDGE IS ASSOCIATED WITH NEGATIVE PATIENT VIEWS}

doi:10.1136/gutjnl-2012-302514b.239

${ }^{1,2} \mathrm{C}$ Selinger, ${ }^{* 2} \mathrm{~J}$ McLaughlin, ${ }^{3} \mathrm{~J}$ Eaden, ${ }^{1} \mathrm{R}$ Leong, ${ }^{2} \mathrm{~S}$ Lal. ${ }^{1}$ Gastroenterology and Liver Services, Concord Hospital and Bankstown Hospital, Sydney, Australia; ${ }^{2}$ School of Translational Medicine, University of Manchester, Manchester, UK; ${ }^{3}$ Gastroenterology, University Hospitals of Coventry and Warwickshire, Coventry, UK

Introduction Enabling women with inflammatory bowel diseases (IBD) to a have successful pregnancy requires complex decisions. This study aimed to assess patients' views on IBD and pregnancy and to correlate them with knowledge.

Methods Female IBD patients aged $18-45$ years were recruited from Australian gastroenterology clinics and private offices. Data were collected on demographics, disease specifics and previous pregnancies. General attitudes were assessed on fertility, medication use, mode of delivery and pregnancy outcomes. Attitudes regarding their personal situation were assessed in participants who had not given birth since their diagnosis of IBD. Agreement was rated on 5 Point Likert scales and knowledge of pregnancy related issues in IBD was assessed by the recently validated Crohn's and Colitis Pregnancy Knowledge Score "CCPKnow". Statistical analysis was performed using Student $t$ test and ANOVA.

Results Of 145 women (median age 32 years, 70\% married) $45 \%$ had Crohn's disease, $45 \%$ ulcerative colitis and 10\% IBD-unclassified. 49 women had successfully delivered children after their diagnosis of IBD. General attitudes: Only 51\% agreed that IBD medication should be continued prior to conception. $68 \%$ agreed with the need for medical therapy for flares during pregnancy, but $24 \%$ felt it more important to tolerate symptoms rather than to have medicines. $36 \%$ of participants believed that any IBD medication is "bad" for unborn children. Patients believed that women with IBD are likely to have a vaginal delivery $(87 \%)$ or a healthy baby $(68 \%)$, but $37 \%$ expected a difficult pregnancy. $70 \%$ thought that women on IBD medication should not breastfeed. Personal attitudes: Of 96 nulliparous women after IBD diagnosis, $46 \%$ were worried about infertility, passing IBD to offspring $(75 \%)$ and $30 \%$ considered not having children because of IBD. $90 \%$ worried about the effect of IBD on pregnancy and $91 \%$ about the effects of pregnancy on IBD. Correlation of attitudes and knowledge: General attitudes that "medication should be stopped prior to conception" ( $p<0.001)$, "pregnant women should avoid all IBD drugs" ( $p<0.001)$, and "put up with symptoms" $(p<0.001)$ were all associated with significantly lower knowledge. Personal attitudes were not associated with knowledge.

Conclusion A significant minority of women felt that IBD medication is harmful to unborn children and women should put up with symptoms. Fear of infertility and concerns about inheritance may explain the extremely high rate of women considering not having children because of IBD. Views contrary to medical evidence were associated with significantly lower knowledge; education and personal counselling should be offered to young women with IBD, particularly those with a low CCPKnow score.

Competing interests None declared.

\section{PM0-240 BETTER DISEASE SPECIFIC PATIENT KNOWLEDGE IS ASSOCIATED WITH GREATER ANXIETY IN INFLAMMATORY BOWEL DISEASE PATIENTS}

doi:10.1136/gutjnl-2012-302514b.240

${ }^{1,2} \mathrm{C}$ Selinger, ${ }^{* 2} \mathrm{~J}$ McLaughlin, ${ }^{3} \mathrm{~J}$ Eaden, ${ }^{1} \mathrm{R}$ Leong, ${ }^{2} \mathrm{~S}$ Lal. ${ }^{1}$ Gastroenterology and Liver Services, Concord Hospital and Bankstown Hospital, Sydney, Australia; ${ }^{2}$ School of
Translational Medicine, University of Manchester, Manchester, UK; ${ }^{3}$ Gastroenterology, University Hospitals of Coventry and Warwickshire, Coventry, UK

Introduction Although inflammatory bowel disease (IBD)-related knowledge empowers patients, it may engender anxiety and impair quality of life (QOL). We aimed to identify predictors of anxiety in IBD and the association with knowledge and disease-related QOL.

Methods Ambulatory IBD patients were recruited from two Australian tertiary hospital clinics and office-based gastroenterologists. Self-administered questionnaire data were collected on demographics and details of IBD, including Crohn's Colitis Association membership status. Disease-related knowledge was assessed using the validated Crohn's and Colitis Knowledge score (CCKnow) and disease related QOL using the short IBD questionnaire (SIBDQ). Anxiety (HADS-A) and depression (HADS-D) were assessed with the Hospital Anxiety and Depression Scores with significance defined as HADS $>10$ and probable disorder as HADS 8-10. Statistical analysis was performed using Student t test and ANOVA.

Results 258 patients $(53.9 \%$ female, median age 47 years) were included. 50 patients (19.4\%) had clinically significant anxiety and $58(22.4 \%)$ had probable anxiety. Age, marital status, highest level of education, employment status, household income, diagnosis (Crohn's vs ulcerative colitis) and duration of disease were all unassociated with higher anxiety levels. Female patients (HADS-A 7.5 vs 6.0 [males], $p=0.003$ ), hospital outpatients (7.8 vs 6.3 [from office based doctors], $\mathrm{p}=0.014$ ) and non-Caucasian patients (7.9 vs 6.4 [Caucasians], $\mathrm{p}=0.037$ ) had significantly higher anxiety levels, while Crohn's Colitis Association members had marginally higher levels of anxiety ( 7.7 vs 6.5 [non-members], $p=0.07)$. Disease related patient knowledge was higher in females (CCKnow 11.3 vs 8.4 [males], $\mathrm{p}<0.001)$, but was not influenced by diagnosis or ethnicity. Anxiety (HADS $\geq 8$ ) was associated with significantly better patient knowledge (CCKnow 10.8 vs 9.3, $p=0.016$ ) and increased depression (HADS-D 6.4 vs 3.1, p<0.001). Disease related quality of life was significantly lower in patients with anxiety (SIBDQ 44.8 vs 57.4 , $\mathrm{p}<0.001)$.

Conclusion Better patient knowledge is associated with higher anxiety levels. Educating patients about their disease and associated risks of surgery, cancer, infertility, etc might trigger anxiety. On the other hand anxious patients might seek out disease related information and hence acquire better knowledge. Our results suggest that an educational intervention may not be suitable to reduce anxiety. Anxiety is common in IBD patients and is associated with depression and significantly impaired quality of life. It may develop as the consequence of diminished quality of life from active IBD, but anxiety itself may lead to impaired quality of life.

Competing interests None declared.

\section{PMO-241 CONVEYING MEDICATION BENEFITS TO ULCERATIVE COLITIS PATIENTS: WHAT THRESHOLDS FOR ADHERENCE ARE APPLIED?}

doi:10.1136/gutjnl-2012-302514b.241

\author{
${ }^{1,2} \mathrm{C}$ Selinger, ${ }^{*}{ }^{1} \mathrm{Y}$ Kinjo, ${ }^{2} \mathrm{~J}$ McLaughlin, ${ }^{3} \mathrm{~A}$ Robinson, ${ }^{1} \mathrm{R}$ Leong. ${ }^{1}$ Gastroenterology and \\ Liver Services, Concord Hospital and Bankstown Hospital, Sydney, Australia; ${ }^{2}$ School of \\ Translational Medicine, University of Manchester, Manchester, UK; ${ }^{3}$ Gastroenterology, \\ SALFORD ROYAL HOSPITAL, Manchester, UK
}

Introduction Non-adherence to maintenance 5-ASA occurs in at least $30 \%$ of ulcerative colitis (UC) patients and is associated with adverse health outcomes and increased healthcare expenditure. Targeted strategies to convey information about benefits of medication to patients may improve adherence. We undertook to discover the preferred mode of information delivery among UC 